\title{
Growth of multi-walled carbon nanotubes on platinum
}

\begin{abstract}
In this paper, Multi-Walled Carbon Nanotubes were grown on a surface of a substrate that consists of a quartz piezoelectric substrate with titanium under layer and platinum electrodes. The Carbon Nanotubes (CNT) was grown using thermal CVD with Iron Nitrate as the catalyst. The growth of the carbon nanotubes was carried out at a temperature of $800^{\circ} \mathrm{C}$ with hydrogen as the process gas and benzene as the hydrocarbon. Characterization of the as grown CNT was done using Scanning Electron Microscope (SEM) and Raman Spectroscopy. The Raman spectroscopy was carried out on a selected area of 100micron by 100 micron and the peaks of the D-band, G-band and the second order modes were observed from the Raman spectra. Image $\mathrm{j}$ image processing software was also used for the extraction of the diameter of the nanotube in which the average diameter was computed to be $46 \mathrm{~nm}$.
\end{abstract}

